\title{
TTR
}

Traduction, terminologie, re?daction

\section{Nicole Brossard traduite en Espagne : re-belle et infidèle? Nicole Brossard Translated in Spain: re-belle et infidèle?}

\section{María Sierra Córdoba Serrano}

Volume 18, numéro 1, 1er semestre 2005

Traductions et représentations : Parcours dans l'espace hispanique II Translations and Representations: Exploring the Hispanic World II

URI : https://id.erudit.org/iderudit/014369ar

DOI : https://doi.org/10.7202/014369ar

Aller au sommaire du numéro

Éditeur(s)

Association canadienne de traductologie

ISSN

0835-8443 (imprimé)

1708-2188 (numérique)

Découvrir la revue

Citer cet article

Serrano, M. S. C. (2005). Nicole Brossard traduite en Espagne : re-belle et infidèle? TTR, 18(1), 91-126. https://doi.org/10.7202/014369ar

\section{Résumé de l'article}

Le contexte socioculturel d'après la Révolution tranquille crée un moment favorable à des pratiques scripturales et traductives féministes, baptisées par Susanne De Lotbinière Harwood " re-belles et infidèles ». Cet article a pour but d'examiner les effets de la réinsertion de ces pratiques de transgression dans un nouveau contexte socioculturel, en l'occurrence le contexte de l'Espagne post-franquiste. Pour ce faire, nous analyserons Barroco al alba (1998), la traduction espagnole du roman de Nicole Brossard Baroque d'aube (1995), seul roman de cette auteure québécoise traduit en Espagne. Nous montrerons que si les « re-belles et infidèles » sont possibles au Canada, c'est parce qu'il y existe un corpus d'oeuvres féministes et donc un contexte favorable à la mise au point de ces stratégies de transgression. Cependant, le contexte espagnol des années 90 n'étant guère réceptif à ce genre de pratiques féministes, Barroco al alba a eu peu de retentissement en Espagne, non seulement dans le sous-champ de grande production (Bourdieu, 1992), mais également dans celui de production restreinte. 


\section{Nicole Brossard traduite en Espagne : re-belle et infidèle?}

\section{María Sierra Córdoba Serrano}

Traditionnellement, la traduction a été envisagée comme une activité transparente, et le sujet traduisant, comme un médiateur neutre se voulant « fidèle " au texte de départ. Cependant, la remise en question post-moderne de certains concepts-clé, comme ceux de " vérité » ou d' " auteur/e », a exercé une influence marquée sur la théorie de la traduction. En se basant principalement sur Derrida, les théoriciens de la traduction ont reconnu que le sens n'est ni stable ni donné par le texte de départ, mais que le sujet traduisant doit l'interpréter à chaque fois. Celui-ci devient dès lors beaucoup plus qu'un simple médiateur : il est responsable d'interpréter l'œuvre d'un auteur pour pouvoir la (re)présenter et l'introduire non seulement dans une nouvelle langue, mais aussi dans une nouvelle tradition, ce qui peut s'avérer problématique. Comme l'affirme Brisset:

La traduction est une activité de compromis entre des langues et des représentations collectives rarement isomorphes, entre des horizons discursifs (cognitifs, épistémiques, esthétiques, traductifs...) qui ne se recoupent jamais complètement, une activité, enfin, dont le sujet négocie et parfois revendique sa propre intervention dans le texte d'autrui. (1997, p. 52)

C’est précisément au cœur de cette intervention dans le texte d'autrui qu'il faut situer les pratiques traductives féministes canadiennes nées à la fin des années 70 et baptisées par Susanne De Lotbinière Harwood " re-belles et infidèles ». Nous verrons que si les re-belles et infidèles sont possibles, c'est grâce à l'existence d'un contexte favorable à la mise au point de ces stratégies de transgression, et nous examinerons ce qui se passe lorsque ces pratiques féministes, scripturales et traductives sont transférées à d'autres contextes socioculturels. Dans le but de 
répondre à cette question, nous analyserons Barroco al alba (1998), la traduction espagnole du roman de Nicole Brossard Baroque d'aube ${ }^{1}$ (1995), seul roman de cette auteure québécoise à avoir été traduit en Espagne.

Nous analyserons dans un premier temps le texte-source, puis le texte cible. Dans le cas du premier, il faut dire que l'auteure de Baroque d'aube présente une double attache, celle d'un sujet doxologique et celle d'un sujet individuel : la première nous permettra de présenter le contexte canadien de la fin des années 70 jusqu'aux années 90, de même que les pratiques d'écriture et de traduction " au féminin " qui se sont développées dans ce contexte. La seconde nous permettra de dresser un bref aperçu de l'œuvre brossardienne afin de bien situer $B A$ dans la période d'utopie lesbienne et des pratiques d'écriture lesbienne " au féminin » qui en découlent. Par la suite, nous passerons à l'analyse même de $B A$, qui prendra la forme d'une analyse thématique.

Par la suite c'est à la traductrice, Pilar Giralt Gorina, que nous nous intéresserons : d'abord, comme dans le cas de l'auteure, en tant que sujet doxologique, ce qui permettra de montrer le contexte socioculturel espagnol du post-franquisme, ensuite en tant que sujet individuel, ce qui nous permettra de situer Barroco al alba dans l'ensemble de sa production et de mieux connaître les visées de sa traduction et d'analyser son rapport au texte-source. Finalement, nous tenterons de montrer que c'est l'appartenance de la traductrice à un contexte particulier, le contexte espagnol des années 90, plutôt que le texte-source lui-même qui s’est avérée déterminante dans la réception du roman en Espagne, ainsi que dans les stratégies traductives adoptées, qui diffèrent grandement de celles de Patricia Claxton, traductrice canadienne-anglaise du même texte.

\section{Nicole Brossard et son contexte socioculturel}

Vers la fin des années 60, soit l'époque de la Révolution tranquille au Québec, les jeunes écrivain.e.s commencent à s'éloigner des discours d'auteurs " révolutionnaires " précédents. À la différence de ces écrivains du milieu des années 60, qui expriment ouvertement l'aliénation culturelle, l'angoisse et la révolte des Québécois, ces jeunes écrivain.e.s s'opposent à l'idée d'une littérature ouvertement politique :

\footnotetext{
${ }^{1}$ Désormais $B A$.
} 
[...] the younger writers were now "colonisés conscients" and could move beyond polemical writing. Moreover, they subscribed to the view that the revolution was being co-opted by academic and middle-class consumer interests which rendered conventional discourse impotent, and forced young writers to develop a critique of it. (von Flotow, 1991, p. 22)

Ainsi, délaissant une littérature " réaliste » et explicitement politique, ces jeunes écrivain.e.s, parmi lesquels Brossard occupe une place centrale, s'intéressent à une littérature qui explore de manière subjective la sexualité et les espaces urbains, mais surtout qui travaille sur le langage en tant que tel (considéré par définition comme politique) et sur sa critique. Cette critique du langage bourgeois sera à l'origine de la critique postérieure, que le mouvement féministe ${ }^{2}$ va promouvoir durant les années 70 et 80.

Dans ce contexte socioculturel, la présence de Nicole Brossard « has been of great significance for all writers interested in la nouvelle écriture [...] [and] it is largely thanks to her efforts and to many new perspectives revealed in her writing that even the most reluctant of critics must admit the existence in Quebec of a distinctive body of feminist literary theory » (Forsyth, 1987, p. 212).

Même si la Révolution tranquille avait rendu possible un grand nombre de changements (la sécularisation de la société québécoise constituant l'un des plus importants), les femmes se sont vues exclues des contributions les plus importantes de cette révolution. D’où l'émergence du mouvement féministe: une multitude de projets féministes de publication, d'information, etc. sont mis en marche pour combattre les attitudes patriarcales des Québécois, qui, malgré le nouveau contexte politique émergeant de la Révolution tranquille, n’avaient pas réellement changé :

Faire la révolution en littérature, c'était un projet de fils élevé contre la mère, c'était une " virilité " à assumer contre et aux dépens de la femme. C'était une naissance, mais une naissance conçue comme rejet, refus et rempart contre la mère trop enveloppante. (Smart, 2003, p. 255)

\footnotetext{
${ }^{2}$ Dans ce travail, quand nous utilisons le terme « féminisme/féministe », nous faisons référence à un féminisme bien précis, le féminisme différentialiste qui se développe dans le contexte québécois des années 70 et 80.
} 
La production littéraire de Nicole Brossard et d'autres écrivaines féministes des années 75 - 80 doit être analysée à la lumière de ce moment de contestation féministe.

Sur le plan littéraire, en particulier, cet esprit contestataire donne lieu à une écriture expérimentale d'avant-garde, qui présente une tendance à la subversion. Il s'agit d'une manière d'aborder l'écriture dans sa matérialité, une écriture ayant pour objectif de créer une modernité dans le champ littéraire québécois. Mais si dans la première étape de son œuvre (le début des années 70), Nicole Brossard aborde l'écriture d'un point de vue formel et cherche la neutralité et l'anonymat " [A writing that] avoids any inscription of the personal, the contextual or the historical » (von Flotow, 1991, p. 30), elle se rend bientôt compte que " "Neutre le monde m'environne neutre”, c’était pour la jeune que j'étais alors, faire preuve d'une bien grande naïveté car, bien sûr, il n’y a de neutre qu'au masculin » (Brossard, 2004, p. 26). Elle commence dès lors à " dé-neutraliser » ses textes, et à y chercher une présence féminine : « La conscience féministe va me déneutraliser, c'est-à-dire me permettre une intégrale présence formelle plutôt que formaliste » (Nicole Brossard en entrevue avec Bonenfant et Gervais, 1985, p. 80). Brossard va donc passer d'une écriture formaliste à une " écriture au féminin ", c'est-à-dire à une écriture certes formaliste, mais politisée et enrichie d'un contenu féministe visant à critiquer le phallocentrisme présent dans le discours et dans l'ordre symbolique.

En partageant leurs recherches sur l'écriture féminine (ou, suivant la dénomination en usage au Québec, l'écriture « au féminin ») ${ }^{3}$ avec les théoriciennes françaises du langage, soit Julia Kristeva, Hélène Cixous et Luce Irigaray, les critiques féministes canado-québécoises, telles que Nicole Brossard, Louky Bersianik, Madeleine Gagnon et France Théoret, se retournent contre la violence du langage phallocentrique. Par leur écriture, elles interviennent au sein même de l'institution patriarcale, de ses symboles et de son imaginaire.

\footnotetext{
${ }^{3}$ France Théoret établit des distinctions plus fermes entre l'écriture féminine (femmes qui écrivent plus ou moins de la même façon que les hommes), l'écriture féministe (écrits de combat, pour changer le sort des femmes), et l'écriture au féminin (écriture qui cherche à réinventer la femme et l'écriture). Ces distinctions sont très courantes parmi les féministes au Québec (King, 2004).
} 
Les critiques féministes canado-québécoises évoquées ci-dessus se rejoignent d'ailleurs dans leur croyance en l'importance du corps dans la libération des femmes vis à vis des attentes patriarcales. Par le fait de valoriser la différence des femmes, l'écriture au féminin, qui implique l'écriture du corps féminin ${ }^{4}$, offre aux femmes la possibilité de devenir agentes de leurs désirs (King, 2004).

Il ne faut pas oublier que la conceptualisation de l'écriture du corps s'inscrit dans un contexte politico-théorique bien concret : un féminisme de la différence s'employant à ériger des frontières conceptuelles entre les deux sexes. De fait, d'autres spectres du féminisme, tels que le féminisme universaliste ou les post-féministes (Dictionnaire critique du féminisme, 2000, pp. 30-35) qualifieront cette démarche d'essentialiste ${ }^{5}$.

Pour ces critiques, la célébration du corps de la femme mène à la création d'un langage spécifiquement féminin. Effectivement, par le fait de prôner la résistance devant le système patriarcal, ces écrivaines soutiennent que l'écriture est nécessairement politique. Ce projet politique englobe aussi ce que l'on a appelé la « traduction au féminin », en tant que forme de réécriture :

Comme l'écriture au féminin, dont elle est tributaire, la traduction au féminin se présente comme une activité politique visant à faire apparaître et vivre les femmes dans la langue et dans le monde. (De Lotbinière-Harwood, 1991, p. 11)

Il s'agit de la traduction des œuvres d'écrivaines féministes québécoises par des écrivaines féministes anglophones. Un aspect très important de ces pratiques d'écriture et de réécriture au féminin est l'intertextualité féministe. On trouve cet intertexte non seulement chez une même auteure, mais entre auteures différentes et il est également présent dans les traductions issues de cette pratique au féminin. Comme l'exprime De Lotbinière-Harwood :

\footnotetext{
${ }^{4}$ À cet égard, Brossard emploie le terme « cortex », composé de corps + texte et servant à exprimer la relation centrale entre le corps et le texte dans l'écriture au féminin.

${ }^{5}$ Pour une excellente analyse de l'essentialisme chez Brossard, voir Ghislaine Boulanger (2004) qui qualifie cet essentialisme de stratégique.
} 
Grâce à l'intertexte féministe, il n'est plus nécessaire de toujours recourir aux ouvrages ou discours érigés en autorité pour puiser nos références et nos exemples, ni pour établir notre crédibilité en nous conformant au code. [...] En circulant dans ce réseau de complicité intertextuelle, on rencontre les mots et les idées d'autres femmes [...]. (1991, p. 58)

Il ne faut pas oublier que cet intertexte féministe se situe dans un contexte socioculturel bien précis. Qu'arrive-t-il alors lorsque ces œuvres sont traduites dans un contexte différent?

Un autre aspect propre au contexte canadien réside dans la double pratique (split practice, von Flotow, 1991) que développent les écrivaines féministes, pratique qui constitue un autre avantage pour la réception et la circulation de ces œuvres et traductions au féminin. Ces écrivaines allient en effet une activité littéraire d'une grande complexité à un activisme sans égal : leurs conférences, leurs articles dans les journaux, leurs pièces de théâtre, etc. ont pour but de préparer les lectrices à la réception de leurs textes littéraires, souvent inaccessibles à la lectrice moyenne. C'est ainsi que Nicole Brossard assumera la cofondation de la revue La Barre du jour (1965-75), organisera des spectacles de jazz et de poésie au Pavillon de la jeunesse lors de l'Expo '67, sera la cofondatrice du journal Les Têtes de pioche (1976-1979), la codirectrice de La Nouvelle Barre du jour (1977-1979) et de la collection « Réelles » (1979-1981). Enfin, sa propre maison d'édition verra le jour en 1982, sous le nom de L'Intégrale, éditrice. Ces activités parmi tant d'autres montrent l'intensité de l'activisme de Nicole Brossard, un activisme qui facilitera la réception de ses œuvres.

Par rapport à l'auteure en tant que sujet individuel, l'œuvre de Nicole Brossard peut se diviser en trois périodes (von Flotow, 1991) soit : le début des années 70 , période pendant laquelle son parcours créatif se caractérise par un intérêt pour la matérialité du langage et les expérimentations scripturales sur la subjectivité et sur la forme. Cette nouvelle écriture représente une réaction aux pratiques littéraires, ouvertement politiques, de la période révolutionnaire et vise à moderniser la littérature québécoise. Une autre des caractéristiques de cette phase est la recherche du " neutre ", de l' " écriture blanche " pour emprunter les mots de Roland Barthes, soit d'une écriture qui élimine de la littérature tout contenu thématique ou tout contexte historique ou personnel. Les produits de cette phase sont, comme le dit Brossard ellemême (1970, citée par von Flotow, 1991, p. 82), « des textes illisibles qui n’ont à peu près d'intérêt que pour celui qui les écrit [...] [pour] 
remettre en question la notion même de la littérature - pour qu'elle devienne l'espace ludique d'une collectivité et non plus le fait morbide de quelques individus ».

La deuxième période, qui se situe vers la fin des années 70, laisse apparaître un glissement du formalisme vers le féminisme. Au cours de cette phase, la nouvelle écriture s'enrichit d'un contenu féministe, donnant lieu au développement de l' « écriture au féminin ». Dans le cadre de cette écriture au féminin, la " grammaire en érection » (La nef des sorcières, p. 130) du patriarcat doit être remplacée par « les mots qui affluent autour du clitoris " (La nef des sorcières, 137). Si l'écriture au féminin et la nouvelle écriture s'intéressent à la matérialité de la langue et à la subversion formelle, leur différence réside dans le fait que l'écriture au féminin ne recherche pas la neutralité. Dans cette deuxième phase, l'espace créé par Brossard n'est pas neutre : c'est un espace au féminin, un espace commun aux femmes.

La troisième période, le début des années 80 , voit se développer une écriture lesbienne au féminin. Le corps lesbien s'inscrit dans l'écriture, mais il va au-delà d'une entité physique : "it is the figure that will subvert constricted knowledge and the stifling discursive order, and widen them into a lesbian utopia " (von Flotow, 1991, p. 88). Durant cette période, Brossard se concentre sur la sexualité lesbienne et le sens lesbien. Elle formule une critique féroce de la maternité dans le cadre du patriarcat, et critique la situation des clans de mères patriarcales qui consacrent leur vie à leur homme, et qui n'ont rien à dire dans leur silence domestique. Brossard propose une nouvelle vision utopique lesbienne très révolutionnaire, dans le cadre de laquelle elle brise la répétition qui transforme toute femme en fille, et développe une utopie des filles-mères lesbiennes. L'écriture devient donc nécessaire pour réinventer le monde. C'est précisément dans cette troisième phase que nous situons Baroque d'aube (1995), même si des éléments appartenant aux phases antérieures s’y trouvent.

Publié en 1995, Baroque d'aube est le huitième ouvrage de fiction de Nicole Brossard, et son avant-dernier roman. Ce roman présente la particularité de remettre en question les convictions sur les rapports entre les images, les mots et la réalité, car " ces réseaux d'informations symboliques, tissés par la culture dominante, relèvent de traditions tenaces et incontournables » (Forsyth, 1985). 
En plus d'être une réflexion théorique, Baroque d'aube est une histoire d'amour: le roman s'ouvre sur un épisode d'amour lesbien entre l'écrivaine Cybil Noland et une violoniste, la Sixtine, qui a lieu dans une chambre de l'hôtel Rafale à Los Angeles. Bientôt, on se rend compte que cet épisode ne correspond pas au récit central du roman, mais qu'il s'agit d'une histoire d'amour inventée par la romancière Cybil Noland, entre elle-même, transposée en personnage fictif, et la Sixtine.

Après cet épisode à Los Angeles, Nicole Brossard reprend le fil conducteur du roman, par la mise en scène d'un navire, le Symbol, qui mène une recherche océanographique au large des côtes argentines. Le personnage de Cybil a été embauché par une océanographe du nom d'Occident DesRives pour rédiger un livre sur la mer, livre qui inclura des photographies prises par Irène Mage, un autre personnage. Cette expédition, dont le point de départ est Buenos Aires, se termine par la mort subite d'Occident. Les cinquante-cinq dernières pages constituent un essai brillant et suggestif sur le métier d'écrivain. Nicole Brossard y aborde l'amitié naissante avec sa traductrice, et l'importance de l'intimité qu'elles partagent.

Pour mieux analyser ce roman, et ce afin d'examiner ultérieurement sa traduction vers l'espagnol, nous nous centrerons sur sa thématique, à savoir d'un côté le projet lesbien-féministe, et de l'autre la recontextualisation du baroque.

Le roman se situe au cœur du projet lesbien-féministe. Du début jusqu'à la fin, le territoire fictif s'oriente autour des expériences et des paroles, des pensées et des rêves de lesbiennes. Selon Brossard, « c’est le débordement, l'élan, l'enthousiasme lesbiens qui avaient permis à la pensée et au discours féministe de se déborder du récit patriarcal, de faire éclater les liens de la double contrainte, de l'insoutenable posture qui était celle du féminin » (Brossard, 1998, p. 54). La femme essentielle - la lesbienne - est pour Brossard la femme idéale, et peupler l'imaginaire de ses désirs et de ses pensées, de ses paroles et de ses rêves, comme elle le fait dans $B A$, est la meilleure façon d'agir pour transgresser le symbolique patriarcal et réinventer le monde : "Une lesbienne qui ne réinvente pas le monde est une lesbienne en voie de disparition » (Brossard, 1998, p. 56). Dans BA, elle présente le désir lesbien et l'acte de faire l'amour entre femmes comme un "vaste espace qui permet de circuler d'un siècle à l'autre » (BA, p. 46), un présent parfait qui transcende l'espace et le temps : «Quand la femme 
avait joui, le ciel, les étoiles et la mer avaient fait synthèse en elle de toute la civilisation de villes » (BA, pp. 15-16). Il s'agit d'un présent qui n'est ni « l'illusion de parfait présent » $(B A$, p. 175$)$ de la réalité virtuelle, ni « le présent du passé » $(B A$, p. 46$)$, que l'on voit lorsque la Sixtine désire connaître le récit du passé de Cybil. Le présent dans $B A$ est associé au corps : " Le corps est vivant, pur présent » $(B A$, p. 23) et ce corps féminin, en particulier, " la peau lesbienne », s'inscrit scripturairement. À la différence d'auteures comme Monique Wittig, pour qui le lesbianisme est politique, Nicole Brossard voit le lesbianisme en termes de désir, en tant qu'un " current of energy that circulates among women » (Parker, 1998, p. 12) ${ }^{6}$.

On verra dans la section consacrée à la traduction l'aspect problématique de la thématique lesbienne pour la réception de $B A$ en Espagne et l'horizon d'attente du milieu espagnol à la fin des années 90.

Nicole Brossard procède parallèlement à une recontextualisation et à une " resignification» du baroque. Le terme baroque (du portugais barroco : roche, perle de forme irrégulière), apparu au $\mathrm{XVIII}^{\mathrm{e}}$, désigne les conventions et les pratiques artistiques en cours vers la fin du $\mathrm{XVI}^{\mathrm{e}}$ siècle et au XVII ${ }^{\mathrm{e}}$ siècle, pratiques qui s'inscrivent dans le contexte de la ferveur religieuse de la Contre-Réforme. Dans son roman, Nicole Brossard inscrit son projet lesbien-féministe dans cette ferveur religieuse en reconstruisant le sujet chrétien en des termes homoérotiques - et plus spécifiquement lesbiens (Moyes, 2000) ${ }^{7}$. En effet, $B A$ reprend le geste de Michel-Ange, et le transforme en rencontre sexuelle de Cybil et la Sixtine. Si dans le récit biblique les sibylles

${ }^{6}$ À cet égard, quelques auteures, telles que Ghislaine Boulanger (2004), qui dans sa thèse doctorale analyse l'essentialisme chez Brossard, affirment qu'étant donné que le féminisme québécois a toujours eu un visage hétérosexuel, Nicole Brossard, dont l’utopie lesbienne n'a pas été bien reçue au Québec, dépolitise le désir lesbien pour continuer à faire partie du mouvement féministe. Selon Boulanger, Brossard dépolitise les causes du désir lesbien en les essentialisant, et ce, pour ensuite politiser ses effets.

${ }^{7}$ En plus de la comparaison entre les lesbiens et les pietàs, Brossard compare aussi les martyrs jésuites et les leathermen. Elle rejette cependant cette dernière image : " L'appropriation de la pietà et le rejet du martyr-devenu leatherman de Cybil en disent long sur ce que la culture lesbienne-féministe sauvegarde et respecte - ce qui y devient "sacré" - et ce qui lui répugne et l'offense " (Moyes, 2000, p. 48). 
occupent une position marginale, pour « la narratrice de Brossard, pour qui le bras droit dénudé de la Sibylle de Cumes est "musclé comme celui de Dieu” (BA, p. 140), les femmes de livres ont un rôle-clé à jouer dans la (représentation de la) Création » (Moyes, 2000, p. 41).

Dans un autre ordre d'idées, l'autre caractéristique du baroque digne d'être soulignée (en raison de sa pertinence pour l'analyse du texte traduit) est son intérêt pour l'allégorie. En fait, comme le remarque Alice Parker (1998, p. 195): « Names in the novel are so pointedly symbolic that the text reads in part like a fable or an allegory ». D’abord, Cybil Noland: son prénom Cybil s'apparente phonétiquement au mot sibylle, et ce choix s'inscrit dans la reconstruction brossardienne de Michel-Ange. Son nom de famille, Noland, No/land cadre bien avec le personnage de Cybil, qui ne veut pas raconter son passé et son histoire à la Sixtine : elle vient de partout et de n'importe où " Je, mon histoire, ce qui existe de moi avant notre rencontre ne vous sont-ils d'aucun intérêt? » (BA, p. 27). Ensuite, le nom de la Sixtine fait référence non seulement au fait qu'elle a l'air d'avoir seize ans « But I am not sixteen » (BA, pp. 16-17), mais aussi à la Chapelle Sixtine. En fait, la rencontre sexuelle entre Cybil et la Sixtine " peut être lue comme une allégorie des liens érotiques qui unissent des lesbiennes de différentes générations, différents discours sur le désir, différentes méthodes de représentation de soi et ainsi de suite » (Moyes, 2000, p. 41).

Quant à l'appellation Irène Mage, qui apparaît parfois comme I. Mage, prononcé comme le mot image, elle fait référence à la culture visuelle actuelle que symbolise ce personnage, en opposition à la culture livresque qui s'intéresse aux mots. Le mot symbolise pour Nicole Brossard la profondeur et l'image, la surface.

Le troisième personnage important est Occident DesRives; elle représente l'Occident, mais un Occident « regenré » en femme :

The oceanographer Occident DesRives is both of that Western tradition and separated from it. Her family name in particular reinforces this double reading, suggesting both the substantive "des rives" (which places her on the edges or margins of any "Occidental" agenda as well as of her own project "sur la mer" and the verb "dériver" (which contains not only the idea of traceable lineage or "derivation" but also that of diversion and drifting). (McPherson, 2000, p. 361) 
D'ailleurs, à l'initiative d'Occident - une femme, ce qui est en soi symbolique -, ces trois femmes entreprennent une expédition à bord du Symbol dans le cadre d'un projet de livre sur la mer. La mer symbolise pour Nicole Brossard l'espace de l'écriture, avec des risques et des possibilités infinies. À bord de ce navire, outre les trois femmes, l'équipage est uniquement constitué d'hommes (le Capitaine Nadeau, padre Sinocchio, le docteur Thomas Lemieux, et un équipage de marins qui regardent des films pornographiques). Ce navire représente donc l'ordre du symbolique masculin, mais la présence féminine compte: "Both women are fatally engaged in bringing emotions and desire into the masculine world of science (not insignificantly "désir" is included, anagrammatically, in the name DesRives " (McPherson, 2000, p. 361).

Nous verrons ultérieurement dans quelle mesure le texte espagnol retient ces allégories.

\section{Pilar Giralt Gorina et le contexte socioculturel de l'Espagne post- franquiste}

La production de la traductrice de $B A$ s'élève à $103^{8}$ traductions. Sa première traduction, recensée dans le Catalogue de la Bibliothèque nationale d'Espagne, date de $1971^{9}$, mais Pilar Giralt Gorina traduit encore à ce jour. Nous pouvons donc situer sa production en tant que traductrice (littéraire principalement) dans les trois dernières décennies $\mathrm{du} \mathrm{XX}^{\mathrm{e}}$ siècle. Cela veut dire qu'elle a connu la dictature franquiste, mais aussi qu'elle est témoin de la transition démocratique. Entre les années 1971 et 1975, elle ne traduit qu'une seule œuvre ${ }^{10}$, le gros de sa production se situant dans les années 80 et 90 . Dans la période postfranquiste, il faut remarquer que, entre 1975 et 1985, même si le régime de Franco était terminé, toute la structure sous-jacente à ce régime politique ne disparaît pas avec son chef, et la production de la

\footnotetext{
${ }^{8}$ Nous avons extrait toute information concernant Pilar Giralt Gorina et sa production du Catalogue de la Bibliothèque nationale d'Espagne, ainsi que des courriels qu'elle a bien voulu nous écrire pour répondre à nos questions.

${ }^{9}$ Il s'agit de Hartley, L. P. El mensajero / Leslie P. Hartley [Pilar Giralt, traducción], Barcelona, Bruguera, [1971].

${ }^{10}$ Ibid.
} 
traductrice se voit affectée par la censure ${ }^{11}$. Il faudra en effet attendre l'arrivée du premier gouvernement socialiste pour que la structure de l'ancien appareil bureaucratique disparaisse complètement.

Enfin, de 1985 à nos jours, il s'agit d'un moment d'ouverture, de modernisation de la société espagnole. Celle-ci, dans les années 90, années de Barroco al alba, parvient, malgré l'hermétisme de la période franquiste, à s'ouvrir progressivement à divers mouvements sociaux, tels que le féminisme ou les mouvements gays et lesbiens.

Il convient cependant de différencier ces deux mouvements et leur acceptation dans le contexte socioculturel espagnol. D’abord, le mouvement féministe existe depuis plus longtemps que le mouvement gay ou lesbien. En fait, pendant la $\mathrm{II}^{\mathrm{e}}$ République espagnole (19311936), le mouvement féministe républicain est considérable (en 1931, les femmes espagnoles obtiennent le droit de vote). Cependant, la victoire franquiste après la guerre civile mènera le féminisme à une impasse. La mort de Franco et le rétablissement de la démocratie ne contribueront guère à augmenter la visibilité du discours féministe. À propos de cette période, Aliaga exprime son mécontentement et sa déception envers les changements sociaux et de mentalité :

la despolitización [...] embargó a España con la llegada de la democracia, que sufrió primero un encantamiento ilusionado, seguido inmediatamente por un desencanto lastimoso, debido, entre otras razones, a la escasa incidencia transformadora en el campo de las costumbres y de la ética (más bien al contrario) que trajo consigo el gobierno socialista. Por otro lado, la invisibilidad del discurso feminista en España impidió la aparición de reflexiones artísticas desde una base teórica fundada en la mujer. (1996, p. 4)

[Avec l'avènement de la démocratie, la dépolitisation [...] a saisi l'Espagne, pays qui est passé d'un jour à l'autre d'un enchantement plein d'espoir à un cuisant désenchantement, attribuable, entre autres, au peu de mesures transformatrices, plutôt le contraire, entreprises par le gouvernement socialiste sur le terrain des coutumes et de l'éthique. D’un autre côté, l'invisibilité du discours féministe en Espagne a

${ }^{11}$ Comme l'affirment Rabadán et Merino (2002, p. 130) : « During the period 1975-1985, which most researchers ignore, cultural products continued to be subjected to the same formal constraints by virtually the same government offices, although their name changed more than once : from censorship to “ordenación” to "calificación”.» 
empêché l'apparition de réflexions artistiques ayant une base théorique fondée sur la femme.] [nos traductions sauf indication contraire]

Bref, la visibilité du mouvement féministe espagnol des années postfranquistes, et ce, même à la fin des années 90, n'est pas comparable au mouvement féministe québécois d'après la Révolution tranquille. D’ailleurs, sur le terrain littéraire, même si le boom de l'écriture des femmes se produit à partir des années 1970 (Francés Díez, 2003), s'échelonnant jusqu'à la fin des années 90 , moment où elle devient de plus en plus visible - pensons à des écrivaines comme Esther Tusquet, Carme Riera, Montserrat Roig, Rosa Montero, Cristina Peri-Rossi (d'origine uruguayenne), etc. -, cette littérature n'est pas aussi expérimentale $^{12}$ et subversive qu'au Québec. Mais surtout, il n'existe pas dans l'Espagne de cette période un sous-champ littéraire féministe ${ }^{13}$ ayant, par exemple, ses propres instances éditoriales et critiques, comme dans le contexte québécois.

Par rapport au mouvement lesbien, la situation est pire encore du fait que le féminisme espagnol est un féminisme hétérosexuel : il faut donc attendre la fin des années 90 pour que le lesbianisme fasse une vraie irruption sur la scène espagnole, et même à ce moment-là, il demeure plus invisible et marginalisé que le mouvement féministe (le lesbianisme étant marginalisé par le propre mouvement féministe). En fait, le silence officiel autour du lesbianisme avant 1975 était total, et si la punition de l'homosexualité était prévue par la " Ley de la peligrosidad y Rehabilitación Social » du 4 août 1970 (Chapitre III, article 6), le lesbianisme n'était pas même mentionné. Comme l’indique Rodríguez (2003, p. 89) : « Sólo a partir de 1975 comienzan a sucederse los actos, los manifiestos y las publicaciones que confieren una identidad y un nombre a las relaciones lesbianas » [Ce n'est qu'à partir de 1975 que commencent à se succéder les actes, les manifestes et les publications qui confèrent une identité et un nom aux relations

12 Il y a toujours des exceptions. Par exemple, Cristina Peri-Rossi fait preuve d'une écriture très expérimentale dans son roman La Nave de los Locos (1984). Ou encore, dans Te deix, amor, la mar com a penyora (1975), Carme Riera montre une certaine expérimentation formelle en utilisant les moyens linguistiques nécessaires pour occulter le sexe des deux femmes protagonistes : elle se servira surtout du pronom catalan " nosaltres ", qui peut référer à deux hommes, deux femmes, à un homme ou à une femme.

${ }^{13}$ À ce propos, voir Isabelle Boisclair (2004). 
lesbiennes]. D’ailleurs, si les revues féministes existaient déjà depuis les années de la transition politique (la première revue féministe, Vindicación feminista, date de 1976), l'apparition de revues lesbiennes aura lieu beaucoup plus $\operatorname{tard}^{14}$. De même, en ce qui concerne la littérature à thématique lesbienne, c'est précisément à la fin des années 90 que cette littérature commence à être visible, mais sa réception était limitée généralement au sous-champ de production restreinte (Bourdieu, 1992). Il faudra attendre dix années pour que cette thématique fasse partie de l'espace des possibles (Bourdieu, 1992) du sous-champ de grande production.

En substance, la présentation générale de ce panorama éditorial et social par rapport au féminisme et au lesbianisme permet de situer la réception de Barroco al alba dans sa société d'accueil. Nous y reviendrons. Pour le moment, retenons que la traduction espagnole se situe à la fin des années 90 , et qu'à cette époque, même si la société espagnole s'ouvre progressivement à une série de mouvements - par exemple gays et lesbiens - comme le montre l'émergence de maisons d'édition et des revues à la thématique homosexuelle, il faudra attendre le début du $\mathrm{XXI}^{\mathrm{e}}$ siècle pour constater l'acceptation dans le sous-champ de grande production d'œuvres littéraires porteuses d'un contenu lesbien et la fondation d'une maison d'édition ${ }^{15}$ et d'une revue spécifiquement lesbiennes. Ainsi donc, au moment de la publication en Espagne de $B A$, la thématique lesbienne se range à un niveau de « semi-clarté " et, dans le cadre de ce développement, on observe une prolifération importante des traductions de romans portant sur ce thème (la production en espagnol étant encore assez réduite) ${ }^{16}$.

${ }^{14}$ La seule revue espagnole destinée exclusivement aux lesbiennes, Femme fatale, est très récente : elle paraît pour la première fois en décembre 2004.

15 Il s’agit d'Ellas, maison d'édition espagnole fondée en 2005. Même s’il y avait d'autres maisons d'éditions à thématique gay, Ellas est la première à se spécialiser exclusivement en thématique lesbienne.

16 Dans le développement de la littérature à thématique lesbienne en Espagne, Palma Borrego (2005) distingue deux phases : la période 1978-1990, qu'elle qualifie de "semi-obscure ", parce que si la thématique lesbienne est présente (dans les œuvres d'auteures telles que Esther Tusquet, Rosa Montero, Montserrat Roig, Mercé Rodoreda, etc.), le modèle lesbien n’est présent que implicitement. La deuxième phase commence à partir des années 90 , où l'on assiste à la parution de romans explicitement lesbiens. Cependant, encore dans cette phase d'après les années 90 , la production en espagnol est très réduite 
Nous avons mentionné précédemment que le Catalogue de la Bibliothèque nationale d'Espagne, attribuait à Pilar Giralt Gorina un total de 103 œuvres ${ }^{17}$ traduites. Une telle production laissait prévoir deux choses: d'une part, que l'on trouverait des renseignements bibliographiques sur elle et d'autre part, qu'il s'agissait d'une traductrice, principalement littéraire et « à temps complet », ce qui est vraiment exceptionnel dans le cas de la traduction littéraire et dans le contexte espagnol.

Notre première prédiction s'est rapidement vue infirmée : nous n'avons absolument rien trouvé sur Internet, pas un seul entretien, aucun article, RIEN. Cependant, après avoir imaginé qu'elle habitait à Barcelone, où se trouvent la plupart des maisons d'édition, nous avons trouvé son adresse et lui avons écrit. Elle a bien voulu nous répondre (très brièvement) et, grâce à ses renseignements et à sa production, nous avons pu obtenir l'information suivante : Pilar Giralt Gorina est bien traductrice, principalement littéraire " à temps complet ». Pour reprendre ses mots ${ }^{18}$ « no soy un personaje digno de mención [formule typique de modestie du sujet traduisant?]; sólo me he limitado a entregarme a mi afición favorita : la traducción ». Curieusement, elle parle de la traduction en tant que afición (passe-temps), plutôt qu'en tant que profession, même si quand nous lui avons posé la question de savoir si elle gagnait sa vie en faisant de la traduction littéraire, elle a répondu par l'affirmative.

Pilar Giralt Gorina a traduit du français, de l'allemand et de l'anglais, mais aussi du russe, du suédois, du norvégien, du danois et de l'italien, ce qui est en soi quelque peu surprenant. Elle a traduit des auteures et des auteurs aussi célèbres que D. H. Lawrence, Doris Lessing, Patrick Süskind, V. Nabokov, Ernest Hemingway, Hermann Hesse, J. D. Salinger, Hans Georg Gadamer et Marlowe, pour n'en citer

comparée à celle d'autres pays. Les traductions y sont donc très nombreuses. La traduction à l'étude se situe précisément dans cette phase. Cependant, cela diffère énormément de la situation actuelle, où l'offre de ce sous-genre est en croissance continue. Voir à ce sujet l'article de Jorge García Velayos, « Literatura homosexual : El libro sale del armario » (2005).

17 Ce nombre n'inclut ni les rééditions d'une même traduction, ni les traductions qui ont été publiées par plus d'une maison d'édition.

${ }^{18}$ Communication personnelle avec Pilar Giralt Gorina du 14 avril 2005. 
que quelques-un(e)s. Elle a traduit pour un grand nombre de maisons d'édition, mais surtout Seix Barral, Plaza\&Janés et Planeta, qui sont très prestigieuses.

Dans le cas de Barroco al alba, ce n'est pas elle qui a choisi de traduire l'œuvre, mais la maison d'édition, Seix Barral, qui lui a proposé la traduction. Ce qui est encore plus révélateur pour notre étude, c'est que Pilar Giralt Gorina avoue n'avoir lu aucun autre livre de Nicole Brossard et le regretter. Cela veut-il dire qu'elle ne connaît ni l'œuvre de Nicole Brossard ni le contexte et les pratiques féministeslesbiennes dans lequel se situe $B A$ ? Peut-être. Pour sûr, elle n'a pas la compétence intertextuelle des traductrices féministes du contexte canadien. Et, bien entendu, à la différence de Patricia Claxton, traductrice de Baroque at Dawn, elle ne compte pas sur la collaboration de Nicole Brossard. Tout cela, comme nous le verrons plus tard, se reflète dans certains de ses choix de traduction et de ses stratégies traductives.

Quand nous l'avons interrogée sur sa relation à l'idéologie féministe du texte de départ, elle a répété qu'elle ne connaissait pas vraiment Nicole Brossard et qu'elle ne pourrait donc pas se définir idéologiquement par rapport à l'idéologie du texte de départ. Nous pourrions donc décrire cette sorte de pratique traductive comme étant non délibérée, et situer Pilar Giralt dans ce que Carol Maier (1998, p. 98) appelle " null strategists " quand elle essaie d'expliquer les différentes manières dont les traductrices ou les traducteurs abordent la question du genre $^{19}$ :

[This 'nul strategy' approach] might be characterized as 'no deliberate approach', and associated with a 'null' strategy or with direct translation of the kind often referred to as 'literal'. This might well be the approach of many translators, both to translating women's fiction and to translation in general. It is the absence of a deliberately formulated method. The absence of an acknowledged approach does not necessarily mean carelessness, however, nor is it purely negative. [...] For such 'null' strategists 'What is a woman?' would not be a question, and there would be no definition of 'woman'. The translator would not feel a need for one.

On saisit le contraste entre la pratique de cette traductrice et l'autre extrême de ce spectre de pratiques traductives, la traduction féministe

${ }^{19}$ Nous employons ce terme dans le sens de gender en anglais. 
ou « au féminin », qui constitue une pratique délibérée et qui présente un projet féministe clairement établi.

Passons maintenant au texte traduit lui-même: comment le rapport au genre de la traductrice, ainsi que son inscription dans un contexte fort différent du contexte canadien ont pu influencer les stratégies traductives adoptées?

La critique littéraire aussi bien que la traduction peuvent être considérées comme des discours de légitimation d'œuvres littéraires (Godard, 1999). Baroque d'aube (1995) a été légitimé par la critique littéraire non seulement au Canada français, mais aussi au Canada anglais, comme l'indique la liste d'articles savants figurant dans notre bibliographie. De même, Baroque d'aube a eu une double légitimation: il a été traduit en 1997 par Patricia Claxton chez McLelland \& Stewart, une maison d'édition canadienne-anglaise, sous le titre Baroque at Dawn. Il s'agit d'une traduction réalisée avec l'appui financier du Conseil des Arts du Canada, et sa publication a également été subventionnée par le Conseil des Arts du Canada ainsi que le Conseil des Arts de l'Ontario. Ce contexte institutionnel diffère énormément du contexte espagnol, où les programmes de subventions à la traduction et à l'édition sont très limités. Soulignons aussi que Baroque at Dawn a été finaliste en 1997 pour le Prix du Gouverneur général, dans la catégorie de la meilleure traduction du français vers l'anglais.

Cependant, malgré toutes ces formes de légitimation, et outre le fait que ce roman répond bien à l'horizon d'attente des milieux de l'avant-garde canadienne-anglaise des années 70 et 80, Baroque at Dawn n'a fait l'objet que de deux comptes rendus dans les journaux anglais $^{20}$ (Godard, 2002), dont un seul commente la traduction (et cela négativement). Selon Godard (2002, p.18), la chose s'explique par le fait que des romans tels que Baroque at Dawn, qui évoquent une esthétique proche du "réalisme magique », esthétique qui se combine à une expérimentation formelle et à une auto-réflexion linguistique, n’ont toujours pas trouvé de ré-écrivain(e)s dans la critique canadienne-anglaise, d'où une limitation de l'œuvre au sous-champ de production restreinte.

${ }^{20}$ Il s'agit du compte rendu de Kate Taylor dans le Globe and Mail (le 3 mai, page D10) et celui de Mary Soderstrom dans Quill \& Quire, un mensuel du monde de l'édition (Godard, 2002). 
Pourquoi donc une maison d'édition aussi prestigieuse que la maison d'édition espagnole Seix Barral prendrait-elle le risque de choisir ce roman et de le traduire dans une société réceptrice dont l'horizon d'attente, y compris dans le sous-champ de production restreinte, ne correspond pas à celui qui est reflété dans $B A$ ?

Pour tenter de répondre à cette question, nous examinerons dans un premier temps le renom de Nicole Brossard (seule écrivaine de la plus récente génération féministe au Québec reconnue au niveau international $^{21}$ ) en Espagne. Dans le contexte péninsulaire, elle n'est pas aussi connue ou célébrée par la critique qu'aux États-Unis, et Baroque d'aube est le seul de ses romans à y avoir été traduit. Le désert mauve a aussi été traduit par Mónica Mansour comme El desierto malva (1996), mais cette traduction a été effectuée et publiée au Mexique, et c'est à peine si elle a été diffusée en Espagne (Suárez Briones, 2001). Par rapport à sa poésie, l'ouvrage Installations (avec et sans pronoms) ${ }^{22}$ vient d'être traduit (en février 2005) en catalan par Antoni Clapés comme Instal.lacions (amb i sense pronoms). Actuellement, c'est en Catalogne précisément qu'elle est un peu plus connue, et en tant que poète plutôt que romancière. À cet égard, elle est décrite dans le peu d'articles que la presse (soit espagnole, catalane ou galicienne) lui consacre comme « una de las figuras más notables de la poesía en lengua francesa hoy » [une des figures les plus connues de la poésie française d'aujourd'hui] (Cinta Montagut, 2005) ou « a autora postmoderne máis importante da literatura canadense » [l'auteure postmoderne la plus importante de la littérature canadienne] (Suárez Briones, 2001). Cependant, hors de la Catalogne, en général, Brossard est presque inconnue sur la scène littéraire espagnole (Montagut, 2005) et, selon certains critiques, « [...] merecería tener una mayor difusión en nuestro país puesto que su lectura no sólo abre nuevos caminos a la reflexión, sino que es también un placer único » [Elle mériterait une plus grande diffusion dans notre pays, car sa lecture non seulement

${ }^{21}$ Brossard a énormément attiré l'attention de la critique américaine. Alice Parker et Karen Gould, parmi d'autres, célèbrent l'utopie lesbienne brossardienne.

${ }^{22}$ Ce livre de poèmes a été publié en espagnol, mais aussi au Mexique. D'autres textes poétiques ont été traduits en espagnol, mais en Argentine et au Mexique : il s'agit de Diario íntimo, El vértigo del proscenio et En el presente de la pulsación. 
ouvre de nouveaux chemins de réflexion, mais procure également un plaisir unique] (Montagut, 2005).

Il est permis de penser que la maison d'édition Seix Barral ait souhaité présenter Brossard sur la scène espagnole, en la faisant connaître en 1998 avec $B A$, pour quelques années plus tard publier une anthologie en langue catalane réunissant des poèmes d'auteurs québécois (incluant Brossard). Suivra une anthologie de poètes catalans, publiée en français en 2002, pour arriver finalement à la publication du livre de poèmes Instal.lacions en catalan (qui avait déjà été publié en espagnol au Mexique). On peut donc voir dans la publication de $B A$ une manière de faire connaître Brossard - qui jouit déjà d'une certaine réputation internationale - en Espagne, et en Catalogne particulièrement. Cet encouragement à fréquenter des auteurs québécois en Catalogne, et, à l'inverse, des écrivains catalans au Québec, doit être replacé dans le cadre des relations entre le Québec et la Catalogne, les deux gouvernements ayant signé, en 1996, une entente cadre de coopération.

Une autre raison qui pourrait contribuer à expliquer la traduction de $B A$ en Espagne serait le fait qu'au moment de la publication du roman, la littérature lesbienne commençait à faire partie de l'espace des possibles (Bourdieu, 1992) du champ littéraire espagnol : on peut donc voir dans cette traduction un renforcement de la visibilité de ce genre de littérature, renforcement qui constitue une prise de position dans cet espace.

Que peut-on dire du public espagnol des années 90 et de son horizon d'attente? L'échec ${ }^{23}$ de $B A$ en Espagne s'explique non seulement en raison de sa thématique lesbienne, mais surtout en raison de l'inscription de ce roman dans des pratiques scripturales d'avantgarde d'une grande complexité. En effet, nous avons mentionné que certaines écrivaines mènent au Québec une double pratique (split practice, von Flotow, 1991), permettant de préparer les lectrices à la réception d'une littérature expérimentale avant-gardiste; or, cette double pratique n'existe pas dans le contexte espagnol. Le seul article que nous avons pu trouver dans des revues (théoriques) féministes sur

${ }^{23} \mathrm{BA}$ appartenant au sous-champ de production restreinte, quand nous parlons d' « échec », nous nous référons à un échec intellectuel, c'est-à-dire, à la quasiinexistence d'un discours critique suscité par Barroco al alba. 
l'écriture et la culture au féminin qui pourrait expliquer l'œuvre de Brossard s'intitule «De radicales a integrales »[De radicales à intégrales] et a été publié dans la revue féministe espagnole Poder y Libertad en 1985. Sur la base des facteurs établis par Jauss (1978) ${ }^{24}$ sur l'horizon d'attente, on peut dire que $B A$ ne correspond pas à l'horizon d'attente du milieu espagnol (au moment de la publication de $B A^{25}$ ), et ce, pour diverses raisons :

a) D'abord, au sujet de la thématique lesbienne (et encore plus, l'homo-érotisation de l'extase religieuse) : il s'agit d'une thématique en émergence, mais assez marginalisée par l'appareil éditorial et critique espagnol. Autrement dit, il n'existe pas dans l'Espagne de la fin des années 90 un sous-champ pour ce genre de littérature avec ses propres instances de production, de légitimation, de consécration et de diffusion.

b) Au niveau formel, ni l'expérimentation formelle ni l'autoréflexion linguistique propre à l'écriture " au féminin », ne correspondent à l'horizon d'attente du public espagnol de la fin des années 90.

L'écart entre l'horizon d'attente des communautés canadoquébécoise et espagnole, ainsi que leurs respectifs contextes socioculturels des années 90, explique principalement l'échec de Barroco al alba en Espagne et son succès ${ }^{26}$ (intellectuel) au Canada. Le contexte socioculturel d'après la Révolution tranquille crée un moment favorable - un Kairos - pour la réception des écrits féministes de l'avant-garde, tandis que la conjoncture socioculturelle espagnole ne se

${ }^{24}$ Jauss (1978 : 49) précise que l’horizon d'attente se compose de trois facteurs principaux : (i) «l'expérience préalable que le public a du genre dont relève le texte littéraire », (ii) « la forme et la thématique des œuvres antérieures » dont l'œuvre nouvelle " présuppose la connaissance ", c'est-à-dire ce que d'autres appelleraient la compétence intertextuelle et (iii) « l'opposition entre langage poétique et langage pratique, monde imaginaire et réalité quotidienne ».

${ }^{25}$ Ce qui ne pourrait pas s'appliquer à la situation d'aujourd'hui.

${ }^{26}$ Il s'agit, bien entendu, de succès intellectuel (Bourdieu, 1992), qui n'est pas mesuré par le tirage, mais par l'importance de l'appareil critique qui se développe autour d’une œuvre. 
montre guère favorable à ce genre de littérature, aux niveaux à la fois thématique et esthétique.

En fait, après avoir consulté les revues littéraires, les journaux espagnols à grande diffusion et quelques journaux plus locaux, nous n'avons trouvé que quelques comptes rendus. Pour les journaux en espagnol, nous avons trouvé un seul - et très court - compte rendu sur Barroco al alba, publié dans El País ${ }^{27}$ (d'ailleurs le seul compte rendu sur Brossard publié dans ce journal). En plus de celui-ci, mais à un niveau plus local, nous en avons trouvé un seul autre publié le 25 septembre 1999 dans La Rioja. Son auteure, María Luisa Lázaro, professeure au Département de philologies modernes, fait les observations suivantes :

No es ésta una novela fácil de leer. [...] No estamos los españoles demasiado acostumbrados a leer literatura canadiense, y casi con toda seguridad éste es el problema. Barroco al alba presenta muchos de los rasgos que caracterizan esta literatura al norte de los Estados Unidos y nos la hacen extraña.

[Ce n'est pas un roman facile à lire. En Espagne, nous n'avons pas l'habitude de la littérature canadienne, et cela pourrait être le problème. Barroco al alba présente beaucoup des traits caractéristiques de la littérature qui se fait au nord des États-Unis et qui nous la rendent bizarre.]

Par la suite, elle décrit ce qu'elle entend par littérature canadienne : « Es la canadiense una literatura escrita mayoritariamente por mujeres que en sus novelas reflexionan sobre la creación literaria, y que al hacerlo reflexionan al mismo tiempo sobre la creación de su propia identidad como mujeres y como escritoras » [La littérature canadienne est une littérature écrite majoritairement par des femmes qui dans leurs romans réfléchissent sur la création littéraire, en même temps que sur la création de leur propre identité en tant que femmes et en tant qu'écrivaines]. Curieusement, on assiste à une féminisation de la littérature canadienne et à l'identification de la littérature canadienne à la littérature féministe québécoise de l'avant-garde ${ }^{28}$. S’opère ainsi une

${ }^{27}$ Voir la bibliographie à la fin de cet article.

${ }^{28}$ Selon ce que nous avons pu observer dans d'autres comptes rendus sur la littérature canadienne ou la littérature québécoise dans la presse culturelle en Espagne, nous ne croyons pas que cela corresponde forcément à la vision générale de la littérature canadienne/québécoise dans ce pays. Selon nous, il 
sorte de confusion métonymique, par le fait de lier la partie, la littérature féministe québécoise, au tout, la littérature canadienne.

Il est également intéressant de constater que María Luisa Lázaro ne mentionne la thématique lesbienne du roman qu'à la toute fin du compte rendu. En revanche, elle se fait insistante sur l'esthétique du roman, à savoir sa fragmentation et sa prose poétique, y voyant un élément potentiellement plus innovateur que la thématique. María Luisa Lázaro termine son compte rendu en exaltant la qualité et le défi que présente la lecture de $B A$, en avertissant toutefois son futur lecteur que ce roman n'est destiné que " para aquellos que ya son aficionados a la literatura canadiense y para los que estén dispuestos a hacer el esfuerzo de aficionarse. Merece la pena, palabra » [pour ceux qui sont déjà de grands amateurs de la littérature canadienne et pour ceux qui sont prêts à le devenir, ce roman vaut la peine. Je vous en donne ma parole]. Elle reconnaît donc qu'il faut, au préalable, une certaine connaissance - une compétence intertextuelle - pour comprendre cette sorte d'écriture. Et elle a tout à fait raison : l'intertextualité est l'une des caractéristiques les plus importantes des œuvres féministes québécoises de l'avantgarde. Pour tout dire, ce compte rendu confirme la non-correspondance de Barroco al alba par rapport à l'horizon d'attente du milieu espagnol, y compris le sous-champ de production restreinte, au sein duquel les agents, autant producteurs que critiques, sont étrangers à l'expérimentation formelle de l'écriture de Nicole Brossard.

Cependant, comme nous l'avons mentionné, Nicole Brossard est plus connue en Catalogne que dans l'ensemble de l'État espagnol, et cela se manifeste dans l'attention qu'on lui porte, à elle et à son œuvre dans le journal catalan Avui : Brossard figure parmi les auteurs québécois qui ont le plus retenu l'attention de ce journal, lequel lui consacre des comptes rendus, des entretiens et des mentions fréquentes. En particulier, le compte rendu consacré à Barroco al alba, intitulé « Nicole Brossard : "Una autora lesbiana té una altra manera d'enfocar l'amor" " [Une auteure lesbienne a une manière autre d'approcher l'amour] (1998), explique la problématique qu'elle explore dans cette œuvre, mais ne fait aucune allusion à l'étrangeté mentionnée dans le compte rendu de María Luisa Lázaro.

s'agit plutôt d'une vision particulière de l'auteure de l'article, qui n'en est pas moins intéressante... 
Nous avons aussi trouvé sur Internet ${ }^{29}$ et sur la couverture de la traduction quelques brefs commentaires sur Barroco al alba. Ces derniers éléments, ajoutés aux articles critiques dont nous venons de parler, ont en commun, outre l'exaltation de la qualité de la prose poétique brossardienne, son caractère innovateur : $B A$ possède " una provocativa capacidad de construcción innovadora » [une capacité provocatrice de construction novatrice] (Barroco al alba, couverture), « una posibilidad de creación innovadora » [une possibilité de création novatrice] (Lázaro, 1999). Plus encore, le roman est « un reto atractivo » [un défi attirant] (Lázaro, 1999), et la traductrice espagnole le décrit en tant que « novedad cautivadora » [une nouveauté captivante] (Giralt Gorina, courriel, 2005). C'est peut-être cette originalité de la prose poétique qui a incité la maison d'édition espagnole Seix Barral à traduire ce roman. En fait, ce caractère novateur cadre bien avec le catalogue de Seix Barral, qui « se ha caracterizado siempre por una gran dialéctica entre la gran literatura y el riesgo por lo nuevo ${ }^{30}$ [a été toujours caractérisé par une dialectique entre la grande littérature et le risque du nouveau]. Parmi les auteurs publiés chez Seix Barral, on trouve des représentants du Nouveau roman, comme Nathalie Sarraute, Monique Wittig et Marguerite Duras, et de la nouvelle écriture, en l'occurrence Nicole Brossard, ce qui confirme un choix pour une littérature en rupture avec le moule du conformisme et du conventionnel.

Regardons maintenant les textes non pas dans le but de commenter chacun des écarts entre l'original et sa traduction, mais afin de relever certains points servant à montrer l'influence d'un contexte socioculturel donné sur l'adoption de certaines stratégies traductives plutôt que d'autres. Il s'agit donc, non pas de critiquer la traduction au nom d'un concept prescriptif du traduire, mais de l'envisager comme

${ }^{29}$ On trouve des commentaires tels que « Barroco al alba, de Nicole Brossard. Ya era hora que alguien tradujera algo de la reina de la escritura lesbiana de qualité, la mítica poetisa, ensayista y narradora québécoise N.B. Relato a varias bandas, con múltiples incursiones en la reflexión sobre el propio acto de escribir » [Barroco al alba, de Nicole Brossard. Il était temps que quelqu'un traduise une œuvre de la reine de l'écriture lesbienne de qualité, la mythique poétesse, l'essayiste et la narratrice québécoise. N.B. récit polyphonique, aux multiples incursions dans la réflexion sur l'acte d'écrire lui-même] http://www.artefinal.com/20anys/guia/sidecar.htm].

${ }^{30}$ http://www.seix-barral.es/editorial.asp. 
« une formation discursive parmi les autres, réglée comme les autres » (Brisset, 1990, p. 25).

Peut-on dégager une stratégie globale de traduction de la version espagnole? Lors de notre examen du rapport de la traductrice au genre, nous avons pu voir que « 'no deliberate approach' [is] associated with a 'null' strategy or with direct translation of the kind often referred to as 'literal' » (Maier, 1998, p. 98). Globalement, en effet, on peut décrire Barroco al alba comme « direct translation of the kind often referred to as 'literal' " (Maier, 1998, p. 98), c'est-à-dire, comme une traduction sourcière. Dans le cadre d'une analyse prescriptive, certains auteurs diraient qu'il s'agit d'une traduction " fidèle " au texte-source, et que c'est donc une bonne (ou mauvaise, diraient les ciblistes) traduction. Cependant, le concept de "fidélité » au texte-source témoigne d'un dogmatisme moralisant et d'une naïveté extrême et il est essentiel ici de considérer, comme nombre de traductologues l'ont déjà dit, que le sujet traduisant part d'abord de l'horizon discursif propre à l'espace culturel récepteur, pour ultérieurement se diriger vers le texte-source.

Dans le cas à l'étude, la connaissance de Nicole Brossard et de toutes les pratiques d'écriture et de traduction " au féminin " par la traductrice et par la société réceptrice est minimale, donc la traductrice essaie de s'éloigner le moins possible du texte-source, de peur, sans doute, de faire dire à Nicole Brossard ce qu'elle n’a pas dit. Étant donné l'absence, en Espagne, d'un contexte de pratiques féministes, scripturales ou traductives, le sujet traduisant et son public ne possèdent pas la compétence textuelle requise. Cette compétence aurait donné à la traductrice une plus grande confiance pour jouer avec la langue, déconstruire le langage et le symbolique patriarcal, bref, s’éloigner du texte de départ et créer une " re-belle et infidèle ». Par la suite, nous illustrerons à l'aide d'exemples cette stratégie globale de la traduction espagnole, que nous comparerons à des moments-clé ${ }^{31}$ avec la traduction anglaise faite au Canada. Notre objectif est de montrer comment certaines stratégies traductives s'expliquent en raison de

${ }^{31}$ Par des « moments-clé », nous nous référons à des passages qui comportent des néologismes ou des termes propres à l'œuvre brossardienne, ou encore des passages obscurs ou des exemples très significatifs, où s’opère un écart marqué quant aux choix de traduction, entre la traduction espagnole et anglaise. Dans ces cas précis, la comparaison avec la version anglaise s’avère révélatrice. 
l'inscription de la traductrice dans des contextes et des paradigmes concrets :

Le premier exemple concerne la traduction du néologisme « vfentre » employé par Brossard :

Vieilles femmes au ventre bas, jeunes lionnes dans la trentaine et d'autres encore plus jeunes, pas formées. Les vfentres me fascinent. Les femmes le portent comme un centre de gravité. Certaines avec fierté comme un projet, d'autres comme un obstacle qui gêne leur démarche. (BA, p. 185)

Mujeres viejas de vientre caído, jóvenes leonas de treinta años y aún más jóvenes sin formar. Los vfientres me fascinan. Las mujeres los llevan como centro de gravedad. Algunas con orgullo, como un proyecto, otras como un obstáculo que dificulta su modo de andar. (BA, p. 143)

Old women with low-slung bellies, young lionesses in their thirties and others even younger and informed. Bellys fancinate me. These women carry a belly like a centre of gravity. Some proudly like a project, others like an obstacle impeding their gait. ( $B D$, p. 181$)$

Le néologisme "vfentre », inventé par l'auteure québécoise, inclut le mot « ventre » et le mot « fente », ce dernier mot étant très utilisé dans d'autres œuvres de Nicole Brossard pour se référer au vagin. La traductrice espagnole, sans la compétence intertextuelle nécessaire pour comprendre ce néologisme, l'a rendu comme " vfientre », ce qui pose problème en espagnol parce que si l'on peut lire dans "vfientre » le mot " vientre ", l'inclusion de la lettre $f$ ne semble pas justifiée à première vue $\mathrm{e}^{32}$ et donc ce choix (que la traductrice n'explique pas) risque de dérouter le lecteur. En revanche, la traductrice du texte anglais, qui connaît bien l'œuvre de Nicole Brossard et a bien saisi la combinaison de ventre+fente, est consciente de l'intraduisibilité de ce néologisme, mais elle adopte une stratégie de compensation en inventant un autre néologisme dans " bellys fancinate », qui inclut le mot « fancy » et le mot « fascinate ». D'ailleurs elle a mis « fancy » en italique pour indiquer au lecteur qu'il ne s'agit pas d'une erreur typographique. Quant à la lettre y du mot « bellys " (à la place de

32 En principe, il nous semble qu'il n'existe aucun mot en espagnol commençant par $f$ pour rendre " fente » dans le sens brossardien. Le mot « fente » pourrait être rendu en espagnol par raja. 
l'orthographe conventionnelle « bellies »), Patricia Claxton ${ }^{33}$ indique qu'elle voulait attirer l'attention du lecteur sur ce mot, comme Nicole Brossard l'avait fait avec le néologisme « vfentre ».

Le deuxième exemple concerne la traduction du mot " délire » employé par Nicole Brossard :

On ne peut pas supplier le désir de nous montrer du doigt le centre, exiger qu'il règle le rythme, ajouter de l'eau à la mer, de la mémoire au corps, du délire au texte. (BA, p. 69)

No se puede suplicar el deseo de enseñarnos el centro con el dedo, exigir que gobierne el ritmo, añada agua al mar, memoria al cuerpo, delirio al texto. (BA, p. 50)

We cannot ask desire to point a finger at the centre for us and at the same time demand that it regulate our rhythm, add water to the sea, memory to our bodies and excitement to what we write. ( $B D$, p. 61$)$

Le terme " délire » fait référence à " un état accidentel entraînant l'abolition de la conscience, et symptomatique de certaines fièvres ou intoxications ${ }^{34}$. Par extension, on l'utilise couramment pour désigner une « exaltation ou une excitation extrême ${ }^{35}$. Chez Nicole Brossard, ce mot veut dire deux choses: soit cette excitation extrême, mais appliquée aux femmes en tant que réponse créative qu'elles ressentent à la lecture des textes d'autres femmes, soit dé/lire (en deux parties) comme une contre-lecture, une lecture qui va à l'encontre de l'ordre patriarcal. Dans le texte anglais, connaissant le sens de l'emploi de ce mot chez Nicole Brossard, la traductrice a choisi de rendre l'un des deux sens du mot dans ce contexte, et, avec excitement et le pronom we (un nous collectif se référant à toutes les femmes), elle rend " délire » dans le sens d'exaltation au moment de l'écriture d'un texte qui sera par la suite lu par d'autres femmes. Elle aurait pu choisir delirium ${ }^{36}$,

${ }^{33}$ Communication personnelle avec Patricia Claxton (le 3 mai 2005).

${ }^{34}$ Le trésor de la langue française informatisé, http://atilf.atilf.fr/tlfi/.

${ }^{35}$ Ibid.

${ }^{36}$ En fait, delirium est le choix de Godard dans sa traduction d'Amantes. Pour rendre l'autre sens de dé-lire en tant que un-read, elle invente le néologisme deliring et écrit reading/deliring pour rendre le jeu de mot brossardien. 
mais ce mot pouvant s'interpréter négativement, dans le sens de confusion mentale, elle élimine l'ambiguïté en employant le mot excitement.

En ce qui concerne le texte espagnol, le mot est rendu par delirio. Le problème est que le principal sens de delirio renvoie d'abord et avant tout à un état de confusion mentale. Étant donné que la traductrice n'inclut aucune note en bas de page pour expliquer le sens de ce mot dans ce contexte, le sens voulu par Brossard passe inaperçu.

Enfin, le troisième exemple concerne la stratégie traductive de la sur-traduction (supplementing, von Flotow, 1991b).

D'abord l'aube. Puis la femme avait joui (BA, p. 13)

Primero el alba. Después la mujer gozó (BA, p. 9)

First the dawn. Then the woman came $(B D, p .5)$

Voilà les deux clauses avec lesquelles débute le roman. On observe que la traduction espagnole laisse implicite ce qui est implicite dans le texte français $^{37}$. Avec ce début, on peut s'imaginer que Nicole Brossard se réfère au moment où la femme atteint l'orgasme; cependant, elle l'exprime de manière poétique et subtile. La traduction anglaise est par contre beaucoup plus explicite dès le début, et certains pourraient dire qu'il s'agit d'une sur-traduction. Néanmoins, on sait qu'il s'agit d'une traduction féministe, d'une " re-belle et infidèle », dont le projet idéologique est " the repossession of the word by women and the naming of the life of the body as experienced by women " (Godard, 1984, p. 14). En effet, l'existence d'un corpus d'œuvres et de traductions féministes rend possible et acceptable l'existence de la traduction anglaise. L'inexistence d'un tel contexte, aussi favorable que le contexte canadien à la mise en marche de ces pratiques traductives transgressives, donne en espagnol : " Después la mujer gozó », plutôt que, par exemple, " Después la mujer se corrió ", qui met l'accent sur l'expression du désir sexuel par les femmes, expression traditionnellement confinée aux hommes.

Tout compte fait, différents procédés de traduction sont à l'oeuvre en fonction des différents contextes et des rapports des

${ }^{37}$ Implicite au moins au début de la lecture et seulement instantanément. Il est évident qu'après quelques lignes, Nicole Brossard explicite qu'il s'agit clairement du moment où la femme atteint l'orgasme. Cependant, il s'agit d'un début assez influent, d'où l'importance de sa traduction. 
traductrices au texte de départ. À cet égard, le texte anglais s'éloigne du texte de départ plus que ne le fait l'espagnol, et présente plus de procédés compensatoires que ce dernier, ce qui s'explique par l'existence de pratiques féministes scripturales propres à l'univers discursif de la version anglaise, mais inexistantes dans l'univers discursif espagnol.

La non-traduction des noms propres du texte-source tient également à cette stratégie globale sourcière que nous avons mentionnée antérieurement. Même si les noms propres ne se traduisent pas en temps normal (sauf pour certaines périodes, qui adaptent même les noms propres), le roman $B A$ peut se lire à la manière d'une allégorie grâce aux noms des personnages. La nature symbolique de ces noms et la possibilité de les traduire sont donc des aspects à considérer. Dans le cas du texte espagnol, les noms ne sont pas traduits, ce qui veut dire qu'une partie importante de l'allégorie brossardienne est perdue. Afin de maintenir l'étrangeté du texte de départ, plutôt que de la domestiquer, tout en reproduisant l'allégorie des noms propres, ces choix de traduction auraient pu être adoptés : pour Cybil Noland, Cybil Sintierra, où l'on garde un nom étranger, Cybil, mais en même temps l'allégorie avec Sin/tierra, nom de famille qui est employé avec une valeur symbolique dans le pseudonyme du célèbre écrivain espagnol José María Blanco White, Juan Sintierra, un nom, donc, connu du public espagnol. Ensuite, Irène Mage aurait pu devenir Irène Magen, en abrégé I.MAGEN : de nouveau, on préserverait ainsi l'étrangeté du nom Irène et l'allégorie du mot imagen. Finalement Occident DesRives deviendrait Occident DesRivas : seraient maintenues l'étrangeté et la métaphore, avec la possibilité de lire en anagramme deriva, qui veut dire " dérive » en espagnol, mais aussi le nom riva, qui n'existe pas en espagnol en tant que tel, mais qui, phonétiquement, se lit comme le mot espagnol riba, qui veut dire « rive ».

La traductrice n'a vraisemblablement pas donné à voir ce symbolisme, ce qui en soi n'a rien d'anormal ou de répréhensible, mais s'inscrit dans un contexte. 
Finalement, l'emploi d'éléments paratextuels ${ }^{38}$, comme les notes en bas de page n'est pas sans intérêt: concrètement, le texte espagnol présente 24 notes, dont la plupart visent à traduire les fragments du texte de départ qui sont en anglais (certains personnages parlant en anglais). La traductrice a maintenu l'hétéroglossie du texte de départ dans le corps du texte espagnol, mais en même temps elle a traduit ces parties en anglais dans les notes en bas de page, sachant que le public espagnol, souvent, ne connaît pas l'anglais. Les autres notes sont culturelles : la traductrice explique par exemple que sundae veut dire « helado con crema, frutas, almíbar y nueces » [Crème glacée avec crème fouettée, fruits, sirop et noix] (BA, p. 79), que djamma est « En árabe, asamblea, reunión de notables » [En arabe, assemblée, réunion de notables] (BA, p. 108) ou que joystick désigne " una palanca de mano » (BA, p. 130).

Paradoxalement, la traductrice n'explique pas dans ses notes les termes brossardiens qui apparaissent tout au long du roman, tels que cortex, délire ou vfentre.

Non seulement la traduction espagnole n'inclut pas de notes pour aider à comprendre ces termes, mais elle n'inclut aucun discours d'accompagnement. Or si ce n'est généralement pas à la traductrice de décider d'inclure ou non des notes (elle n'est payée que pour la traduction), nous nous demandons si elle aurait pu insister un peu plus sur l'inclusion de quelques notes pour expliquer certains termes ${ }^{39}$. Étant donné que Nicole Brossard n'est guère connue dans le milieu espagnol, il aurait été très utile d'inclure un discours d'accompagnement visible, comme une préface ou une postface, visant à présenter Nicole Brossard et son œuvre à la communauté réceptrice et, si possible, à expliquer les stratégies traductives adoptées pour la traduction de $B A$ en espagnol.

L'inclusion d'éléments paratextuels du type de ceux que Massardier-Kenney (1997, pp. 58-65) décrit en termes de " authorcentered » et de " translator-centered » aiderait à la réception du

\footnotetext{
${ }^{38}$ Nous nous référons à la théorie du paratexte de Gérard Genette (1987). Le paratexte se définit par les éléments d'accompagnement d'un texte, c'est-à-dire la présentation extérieure du livre, le nom de l'auteur, le titre, le «prière d’insérer », les dédicaces, les épigraphes, les préfaces, les postfaces, les intertitres ou encore les notes.

${ }^{39}$ Nous sommes conscientes que les contraintes éditoriales priment parfois sur la volonté du traducteur.
} 
roman. De plus, n'est-ce pas le rôle de la traductrice/du traducteur, en tant que sujet chargé d'établir le rapport à l'Autre, d'insister sur l'inclusion d'un paratexte? Revendiquer un statut pour le sujet traduisant n'est-ce pas aussi assumer la responsabilité d'introduire et de (re)présenter l'auteure traduite et son contexte dans la société d'accueil? Mais on a vu au début de l'article la posture effacée de la traductrice...

Dans le cadre de cette responsabilité représentationnelle du sujet traduisant, nous terminerons sur un dernier aspect du texte espagnol : le maintien de l'hétéroglossie du texte original. Dans le texte-source en français, Nicole Brossard donne la parole à certains personnages en anglais. Cette hétéroglossie est sélective, et remplit une fonction particulière, ce que la traductrice espagnole a saisi. Le mélange de l'anglais et du français dans $B A$ comporte une valeur sociologique importante (Godard, 2002) : est ainsi mise en évidence la réalité sociale et historique des milieux lesbiens montréalais caractérisés par le bilinguisme. Même si dans le roman il est vrai que la question de la différence sexuelle prime sur celle de la différence linguistique, le maintien de l'hétéroglossie compte davantage qu'une certaine « fluidité » de la traduction.

De plus, le but de la traduction de Baroque d'aube n'est pas seulement de véhiculer un savoir référentiel sur la sexualité lesbienne en général, mais de situer cette sexualité dans un contexte historique et social particulier, le contexte québécois. Si les protagonistes sont lesbiennes, elles font également partie d'une société, la société québécoise, au sein de laquelle le mélange linguistique témoigne d'un rapport de forces. Sur ce point, Pilar Giralt Gorina fonde une sorte d'éthique de la différence culturelle par sa tentative de ne pas décontextualiser ou déterritorialiser la traduction, en montrant clairement les marques de sa réénonciation.

Somme toute, le texte espagnol alterne entre une éthique de la différence culturelle et une ouverture idéale à l'altérité du texte-source, avec des moments où le rapport à l'Autre n'est guère exemplaire. En fait, ce comportement reflète tout simplement l'ambiguïté inhérente à tous les rapports d'altérité. Cette sorte de lutte, ou de relation amourhaine, est une réalité à laquelle on ne peut échapper, même dans le meilleur des cas, et ce, même quand on essaie d'aborder l'Autre en tant qu'Autre, et non en tant que Même. Barroco al alba ne fait pas 
exception à cette réalité, qu'on pourrait à la rigueur qualifier d'axiomatique.

Tels sont quelques-uns des effets de la réinsertion des pratiques scripturales et traductives féministes dans un nouveau contexte, en l'occurrence, la recontextualisation des pratiques féministes canadiennes, dans lesquelles se situe Baroque d'aube, à l'intérieur du contexte espagnol. Si les « re-belles et infidèles » sont possibles au Canada, c'est parce qu'il existe un corpus d'œuvres féministes et donc un contexte favorable à la mise au point de ces stratégies de transgression (Lotbinière-Harwood, 1991).

Cependant, le contexte espagnol des années 90 n'étant guère réceptif à ce genre de pratiques féministes, Barroco al alba a eu peu de retentissement en Espagne. Serait-ce, comme l'affirme Fefa Vila (2001), du fait que l'Espagne a longtemps accusé un retard par rapport à ses voisins occidentaux, non pas sur la question de la reconnaissance des droits, mais sur celle des changements sociaux qui encouragent la diversité des pratiques sexuelles et des styles de vie? Faut-il penser que le changement ne s'est produit que très récemment au sein de la société espagnole? Le contexte espagnol actuel, maintenant plus que jamais, constituerait une conjoncture propice à la réception d'œuvres féministes québécoises. En effet, après les attentats du 11 mars 2004, le peuple espagnol a vécu un grand nombre de changements : un nouveau gouvernement, de nouvelles lois (la loi sur le mariage homosexuel est approuvée en Espagne en juillet 2005) et, surtout, une tentative de révolte socio-sexuelle, de toute évidence accompagnée d'une réactivation des idées féministes, qui circulent dorénavant partout dans le pays. Les citoyens ont dénoncé la manipulation de l'information par le gouvernement d'Aznar, de même que ses politiques néolibérales et ultra-catholiques. Ce virage historique a secoué les traditions, et, suite aux événements mentionnés, on a commencé à parler d'une « Révolution tranquille » espagnole (L’Actualité, p. 24, mars 2005) ou d'une « sexual and social revolution » en Espagne (New York Times, p. A4, le 31 mars 2004).

En définitive, espérons que cette conjoncture actuelle favorable permettra la mise en place de stratégies féministes de transgression qui, comme dans le contexte canadien, aideront la traductrice à passer du statut de deuxième auteure à celui d'auteure, et la traduction de deuxième texte à celui de texte.

Université d'Ottawa 


\section{Références}

ALIAGA, J. (1996). « ¿Existe un arte queer en España? ». Acción Paralela, 3, http://www.accpar.org/numero3/queer.htm.

ARSENAULT, M. (mars 2005). " L’Espagne se dévergonde ». L'Actualité, p. 24.

B. G. (1998). « En su libro 'Barroco al alba', Nicole Brossard ofrece la exploración literaria de una historia ». El País, le 19 juillet.

BOISCLAIR, I. (2004). Ouvrir la voie/x. Le processus constitutif d'un sous-champ littéraire féministe au Québec (1960-1990). Québec, Nota bene.

BONENFANT, J. et A. GERVAIS (1985). « Ce qui pouvait être ici une avant-garde ». Entretien avec les membres fondateurs de La barre du jour dans Voix et Images, X, 2, pp. 68-85.

BOULANGER, G. (2004). Feintes, essences et mimésis chez Nicole Brossard, Patrick Imbert et Marie-Claire Blais. Thèse de doctorat, Université d'Ottawa.

BOURDIEU, P. (1992). Les règles de l'art. Genèse et structure du champ littéraire. Paris, Seuil.

BRISSET, A. (1997). « La traduction, modèle d'hybridation de cultures? ». Carrefour, 19, 1, pp. 51-69.

- (1990). Sociocritique de la traduction. Théâtre et altérité au Québec (1968-1988). Québec, Le Préambule.

BROSSARD, N. (2004). Écrire l'horizon du fragment. Québec, Éditions Trois-Pistoles.

- (1998). «Écriture lesbienne : écriture de marque ». Les études gay et lesbiennes, pp. 51-56.

- (1998). Barroco al alba. Traducido por Pilar Giralt Gorina. Barcelona, Seix Barral. 
- (1997). Baroque at Dawn. Translated by Patricia Claxton. Toronto, McClelland \& Stewart.

- (1995). Baroque d’aube. Montréal, L’Hexagone.

-(1976). La nef des sorcières. Montréal, L’Hexagone.

CASTELLS, A. (1998). « Nicole Brossard: "Una autora lesbiana té una altra manera d'enfocar l'amor” ». Avui, le 13 juillet.

Dictionnaire critique du féminisme (2000). Sous la coordination de Helena Hirata, Françoise Laborie, Hélène Le Doaré et Danièle Senotier. Paris, Presses de l’Université de France.

FORSYTH, L. (1987). " Nicole Brossard and the Emergence of Feminist Literary Theory since 1970 ». Gynocritics/La gynocritique. Ed. Barbara Godard. ECW Press, pp. 211-222.

- (1985). Compte rendu sur Baroque d'aube en ligne: http://felix.cyberscol.qc.ca/LQ/auteurB/brossa_n/Baroq_br.html.

FRANCÉS DÍEZ, M.A. (2003). « La represa de la paraula: influència del feminisme em la literatura catalana actual ». Feminismo/s, 1, pp. 117-134.

GARCÍA VELAYOS, J. (2005). « Literatura homosexual: el libro sale del armario ». Delibros, 186, pp. 40-45.

GENETTE, G. (1987). Seuils. Paris, Seuil, collection « Poétique ».

GODARD, B. (2002). « La traduction comme réception : les écrivaines québécoises au Canada anglais ». TTR, 15, 1, pp. 65-98.

- (1999). « Une littérature en devenir: la réécriture textuelle et le dynamisme du champ littéraire. Les écrivaines québécoises au Canada anglais ». Voix et images, XXIV, 3, pp. 495-527.

- (1984). "Translating and Sexual Difference ». Resources for Feminist research, 13, 3, pp. 13-15.

JAUSS, H. R. (1978). Pour une esthétique de la réception. Paris, Gallimard. 
KING, A. (2004). Figures de subversion: anorexie, prostitution et écriture dans Putain de Nelly Arcan. Thèse de Maîtrise (unpublished), Queen's University.

LÁZARO, M. L. (1999). « Barroco al alba ». La Rioja, le 25 septembre.

LOTBINIÈRE-HARWOOD, S. De. (1991). Re-belle et infidèle : la traduction comme pratique de réécriture au féminin/The body bilingual : translation as rewriting in the feminine. Montréal/Toronto, les éditions du remue-ménage/Women's Press.

MAIER, C. (1998). «Issues in the Practice of Translating Women's Fiction ». Bulletin of Hispanic Studies, LXXV, pp. 95-108.

MASSARDIER-KENNEY, F. (1997). « Towards a Re-definition of Feminist Translation Practice ». The Translator, 3, 1, pp. 55-70.

MCPHERSON, K. S. (2000). "Writing the Present in Nicole Brossard's Baroque d'aube ». American Review of Canadian Studies, 30, 3, pp. 361-383.

MERINO, R. et R. RABADÁN (2002). " Censored Translations in Franco's Spain: The TRACE project-Theatre and Fiction (EnglishSpanish) ». TTR, 15, 2, pp. 125-152.

MONTAGUT, M. CINTA (2005). " Nicole Brossard : feminismo y escritura. Instal.lacions (amb i sense pronoms) ». En ligne: http://www.barcelonareview.com/47/s resen.html\#1.

MOYES, L. (2000). "Rien de sacré: Baroque d'aube de Nicole Brossard aux limites des discours lesbiens-féministes sur la sexualité ». International Journal of Canadian Studies/Revue internationale d'études canadiennes, 21, pp. 35-63.

La nef des sorcières (1992). Collectif de Luce Guilbeault, Marthe Blackburn, France Théoret, Odette Gagnon, Marie-Claire Blais, Pol Pelletier et Nicole Brossard. Montréal, L’Hexagone.

PALMA BORREGO, M. J. (2005). « La literatura lesbiana española: un lugar casi desierto». En ligne:

http://www.lesgou.com/articulos/articulos/index_lesespana.htm. 
PARKER, A. (1998). Liminal Visions of Nicole Brossard. New York, P. Lang.

RODRÍGUEZ, M. P. (2003). « Crítica lesbiana: lecturas de la narrativa española contemporánea ». Feminismo/s, 1, pp. 87-102.

SCIOLINO, E. (mars 2004). «A New Future for Spain : Call it Social Socialism ». New York Times, p. A4.

SMART, P. (2003). Écrire dans la maison du père: L'émergence du féminin dans la tradition littéraire du Québec. Montréal, XYZ éditeur.

SUÁREZ BRIONES, B. (2001). « Escribir en femenino supón tremer ». Entretien entre Nicole Brossard et Beatriz Suárez Briones. En ligne: http://www.vieiros.com/noticia.asp?Ed=18\&N=16366.

VILA, F. (2001). « Genealogías del lesbianismo : historias de mujeres y literatura ». En ligne : http://www.hartza.com/orlando.html.

VON FLOTOW, L. (1991). Quebec Feminist Writing : Integrating the Avant-Garde and the Political in the Works of Nicole Brossard and France Théoret. Thèse de doctorat publiée, Université de Michigan, Ann Arbor.

- (1991b). " Feminist translation: Contexts, Practices and Theories ». TTR, 4, 2, pp. 69-84.

RÉSUMÉ : Nicole Brossard traduite en Espagne : re-belle et infidèle? - Le contexte socioculturel d'après la Révolution tranquille crée un moment favorable à des pratiques scripturales et traductives féministes, baptisées par Susanne De Lotbinière Harwood « re-belles et infidèles ». Cet article a pour but d'examiner les effets de la réinsertion de ces pratiques de transgression dans un nouveau contexte socioculturel, en l'occurrence le contexte de l'Espagne post-franquiste. Pour ce faire, nous analyserons Barroco al alba (1998), la traduction espagnole du roman de Nicole Brossard Baroque d'aube (1995), seul roman de cette auteure québécoise traduit en Espagne. Nous montrerons que si les "re-belles et infidèles " sont possibles au Canada, c'est parce qu'il y existe un corpus d'œuvres féministes et donc un contexte favorable à la mise au point de ces stratégies de transgression. Cependant, le contexte espagnol des années 90 n'étant 
guère réceptif à ce genre de pratiques féministes, Barroco al alba a eu peu de retentissement en Espagne, non seulement dans le sous-champ de grande production (Bourdieu, 1992), mais également dans celui de production restreinte.

ABSTRACT: Nicole Brossard Translated in Spain: re-belle et infidèle? - The socio-cultural context following the Quiet Revolution has created a favorable moment for the implementation of feminist writing and translation practices, termed " re-belles et infidèles » by Susanne De Lotbinière Harwood. The purpose of this article is to examine the effect of the reinsertion of these transgressive practices in a new context, namely, post-Franco Spain. With this in mind, we will analyze Barroco al alba (1998), the Spanish translation of Nicole Brossard's novel Baroque d'aube (1995), the only one by this author that has been translated in Spain. We will show that " re-belles et infidèles " practices are possible because there already exists a corpus of feminist writing and thus a favorable context for the implementation of these transgressive practices. Nevertheless, the Spanish context of the 90s not being receptive to these feminist practices, Barroco al alba has gone unnoticed in Spain, not only in the subfield of large-scale cultural production (Bourdieu, 1992), but also in the restricted production subfield.

Mots-clés : traduction féministe, Nicole Brossard, Barroco al alba, réception, Espagne post-franquiste.

Keywords: feminist translation, Nicole Brossard, Barroco al alba, reception, post-Franco Spain.

María Sierra Córdoba Serrano : École de traduction et d'interprétation, Université d'Ottawa, Pavillon des arts, 70, rue Laurier Est, Ottawa (ON) K1N 6N5

Courriel : mcord059@uottawa.ca 